\section{Chromatographic Separation of Digitalis Glycoside}

SEVERAL of the constituents of Digitalis purpurea have been separated by paper partition chromatography, using ethylene glycol as the stationary phase and chloroform saturated with ethylene glycol as the moobile phase.

This system offers an advantage over the chloroform/formamide system used by Jensen ${ }^{1}$ since the primary $A$ and $B$ glycosides as well as the secondary glycosides are separated before the solvent front reaches the bottom of the paper. Furthermore, any ethylene glycol left in the paper after drying will not react with the alkaline reagents, such as $3: 5$ dinitrobenzoate, commonly used to estimate the digitalis constituents, whereas formamide gives a marked reaction.

The toluene/propylene glycol system described by Zaffaroni $^{2}$ and used by Heftmann and Levant ${ }^{3}$ for separating cardiac glycosides was tried but did not separate the digitalis constituents sufficiently for quantitative work.

In general, chloroform is preferable to toluene as the mobile phase for the separation of digitalis glycosides ; propylene glycol and chloroform, however, are miscible and therefore unsuitable. On the other hand, ethylene glycol is relatively immiscible with chloroform and this combination has proved satisfactory. The method used is as follows.

Strips of Whatman No. $3 M M$ chromatograph paper $7.5 \mathrm{~cm} . \times 55 \mathrm{~cm}$. are passed through a 30 per cent $\mathrm{v} / \mathrm{v}$ solution of ethylene glycol in methanol and blotted between folds of Whatman No. 1 paper. Solutions of the glycosides and aglycones in ethanol are applied to the starting line and the strips hung overnight in a chamber saturated with chloroform. Development is then commenced by allowing the mobile phase, chloroform saturated with ethylene glycol, to pass down the paper strips. There is a tendency for ethylene glycol to separate from the mobile phase in the trough and saturate the end of the paper, thus considerably slowing the rate of development. This may be prevented by placing a fold of dry Whatman No. $3 M M$ paper over this end of the strip before placing in the trough.

In the developed chromatograms each of the constituents occupies a small compact area with little tailing even when loads of $0.5 \mathrm{mgm}$. are chromatographed. Approximate $R_{F}$ values are as follows :

$\begin{array}{llll}\text { Desacetyldigilanid } B & 0.05 & \text { Gitoxin } & 0.80 \\ \text { Desacetyldigilanid } A & 0.20 & \text { Digitoxigenin } & 0.90 \\ \text { Gitoxigenin } & 0.55 & \text { Digitoxin } & 0.95\end{array}$

Satisfactory quantitative recovery of several of the constituents has been obtained from the developed chromatograms, after removal of the ethylene glycol by heating for two days in a current of air at a temperature of $30^{\circ} \mathrm{C}$. The glycosides present in alcoholic extracts of powdered digitalis leaves have also been separated and estimated.

The same solvent system has been used in preliminary experiments for separating mixtures of coumarin and related compounds, prior to testing for root growth inhibition. The degree of separation was of the same order as that obtained with systems containing butanol, acetic acid and water ${ }^{4}$. Chromatograms containing butanol and acetic acid in the mobile phase, after drying at room temperature for five hours, retained sufficient of the organic solvents to suppress completely root growth in cress seedlings. On the other hand, the amount of ethylene glycol remaining in chloroform/glycol chromatograms, similarly dried, did not cause significant inhibition of root growth.

G. J. RIGBY

D. M. BeLLIS

Department of Pharmacy,

The University, Manchester.

1 Jensen, K. B., Acta Pharmacol. et Toxicol., 8, 99 (1953).

2 Zaffaroni, A., Burton, R. B., and Keutmana, E. H., Science, 111, 6 (1950).

${ }^{3}$ Heftmann, E., and Levant, A. J., J. Biol, Chem., 194, 703 (1952)

"Swain, T., Biochem. J., 53, 200 (1953).

\section{Tumour-inhibiting Properties of Potassium Iodide Solution containing Potassium lodate}

F. B. Oberhauser et al. ${ }^{1}$ have recently published results of experiments in which they tried to influence the growth of a rat sarcoma by oral treatment of the animals with solutions of potassium iodate in potassium iodide.

The surprising results obtained by these authors seemed to justify a repetition of the experiments using another strain of rats and a sarcoma obtained from another source.

We used male Wistar rats aged $3-3 \frac{1}{2}$ months and an average weight of $200 \mathrm{gm}$. These were implanted with 5-10 mm. ${ }^{3}$ samples of rats' Jensen sarcoma sub. cutaneously in the back. (We owe this tumour to Prof. Lettre from the Cancer Research Institute of the University of Heidelberg.) One hundred animals used in these preliminary experiments were divided into three equal groups. The animals of the first group were implanted with the tumour only, whereas those of the second group were treated only with the solution used by Oberhauser et al. by stomach tube. The animals of the third group were treated with $0.2 \mathrm{ml}$. of this solution three times daily for 20 days preceding the tumour implantation. Those of the fourth group received the same treatment 10 days before and 10 days after the tumour implantation. Finally, the fifth group were treated with $0.2 \mathrm{ml}$. of the solution for 20 days after the implantation.

The results can be summarized as follows. All animals of the first group, with the exception of two which died from a wound infection on the third and fourth day respectively, died within about 30 days. The majority of the animals in group 2 died from diffuse hæmorrhagic gastritis. In these, numerous erosions of the gastric mucous membrane were found and in some cases the stomach was filled with blood Some animals died from bronchopneumonia. On the thirty-fifth day of the treatment only six survived; these too had lost much weight and were apathetic and without appetite. Most animals of group 3 died before the implantation of tumours, partly from hæmorrhagic gastritis and partly from bronchopneumonia, others from unknown reasons (some of the dead animals had been mutilated by their litter mates). Those animals which survived the pretreatment died from their tumours after an average of 30 days. The experiments of groups 4 and 5 had an analogous result.

The rats used in our experiments can therefore be said to have sustained great damage when treated with the solution described by Oberhauser et al. in the way and in the same quantity used by these authors, causing the death of the majority of the animals. Those animals which survived the treat. 